\title{
Chemical Potential vs. Gibbs Free Energy Relationship by Redlich and Kister and the Redlich-Kister Polynomial
}

\author{
H. Näfe \\ Universität Stuttgart, Institut für Materialwissenschaft \\ Heisenbergstraße 3, 70569 Stuttgart, Germany \\ E-mail: naefe@imw.uni-stuttgart.de
}

\begin{abstract}
The relationship of Redlich and Kister between the chemical potential and the molar Gibbs free energy of a multicomponent mixture proves to be in contradiction with both fundamental concepts of chemical thermodynamics and mathematical principles. Hence, it is invalid. This invalidity corresponds with the thermodynamically inadequate representation of the prevalent Redlich-Kister polynomial for the mole fraction dependence of the excess Gibbs free energy of a mixture.
\end{abstract}

Keywords: Redlich-Kister polynomial; Euler's homogeneous function theorem; chemical potential; Gibbs free energy

\section{Introduction}

The relationship due to Redlich and Kister [1] between the chemical potential of a component of a mixture and the Gibbs free energy of the same mixture, or between the respective excess quantities, is widespread in chemical thermodynamics. It is based on the assumption that, mathematically, the mole fractions of all $N$ components comprising the mixture represent independent variables This actually opposes the thermodynamic reality since at least one of these mole fractions can always be expressed by means of the sum of all others. Nevertheless, recently it has been shown by Näfe [2] that Redlich and Kister's relationship proves to be mathematically equivalent to those relationships that refrain from assuming total independence of all mole fractions and instead take the thermodynamic facts properly into account. This quite paradoxical situation with apparently equivalent results despite apparently conflicting assumptions, which has persisted in the literature for about 65 years, calls for clarification.

In the following, the problem is resolved by demonstrating that the relationship of Redlich and Kister is at variance with both thermodynamics and mathematics and that this is strongly related to the representation of the Redlich-Kister polynomial that is abundantly often employed in chemical thermodynamics for the description of multicomponent systems.

\section{General Relationships}

The excess Gibbs free energy $g^{E}$ is an extensive quantity depending on the pressure $p$ and the temperature $T$ as well as on all mole numbers $n_{i}$ of the thermodynamic system under consideration with $i$ ranging from 1 to $N$ :

$g^{E}=f\left(p, T, n_{l}, \ldots, n_{N}\right)$

Division of $g^{E}$ by the total number of all moles comprising the particular system yields the molar Gibbs free energy $G^{E}$ as an intensive quantity:

$$
G^{E}=\frac{g^{E}}{\sum_{i=1}^{N} n_{i}}
$$

In view of Eqs. (1) and (2), $G^{E}$ can be represented by the same functional dependence as defined by Eq. (1):

$G^{E}=f\left(p, T, n_{l}, \ldots, n_{N}\right)$

It will become obvious from the paragraph after the next that the extensive character of $g^{E}$ is in accordance with Euler's homogeneous function theorem and thus with the relationship:

$$
g^{E}=\sum_{i=1}^{N} n_{i} \cdot \mu_{i}^{E}
$$

In Eq. (4) the excess chemical potential $\mathrm{m}_{k}^{E}$ is defined as partial derivative of $g^{E}$ with respect to the number of moles of the $k$-th sort upon holding constant all other mole numbers $n_{j}$ :

$\mu_{k}^{E}=\left(\frac{\partial g^{E}}{\partial n_{k}}\right)_{p, T, n_{j}}$

By substitution of Eq. (2) into Eq. (5) it can be shown that:

$$
\mu_{k}^{E}=G^{E}+\sum_{i=1}^{N} n_{i} \cdot\left(\frac{\partial G^{E}}{\partial n_{k}}\right)_{p, T, n_{i}}
$$

Instead of the mole number the respective mole fraction $x_{k}$ can be used as a composition variable. The definition is:

$x_{k}=\frac{n_{k}}{\sum_{i=1}^{N} n_{i}}$ 
This definition implies that all $x_{i}$ are not independent of each other since:

$$
x_{k}=1-\sum_{i=1, \neq k}^{N} x_{i}
$$

\section{Relationship by Redlich and Kister}

As mentioned earlier [2] there is merely conjecture about the details of deriving the relationship as the authors did not at all dwell on that. It must be assumed, and this is confirmed by a later approach [3], that the starting point is the representation of the excess Gibbs free energy as a function of the intensive variables $p, T$ and $x_{i}$ with $i$ ranging from 1 to $N$ :

$$
G_{R K}^{E}=f\left(p, T, x_{1}, \ldots, x_{N}\right)
$$

In order to distinguish this functional dependence from the definition (3) the index RK is used hereinafter. According to Eq. (9) the total differential of $G_{R K}^{E}$ under isobaric and isothermal conditions reads:

$$
\left(d G_{R K}^{E}\right)_{p, T}=\sum_{i=1}^{N}\left(\frac{\partial G_{R K}^{E}}{\partial x_{i}}\right)_{p, T, x_{j}} \cdot d x_{i}
$$

Differentiation of Eq. (10) with respect to the mole number of one arbitrary sort of components of the system, e.g. $n_{k}$, upon holding all other components' mole numbers constant leads to:

$$
\left(\frac{\partial G_{R K}^{E}}{\partial n_{k}}\right)_{p, T, n_{j}}=\sum_{i=1}^{N}\left(\frac{\partial G_{R K}^{E}}{\partial x_{i}}\right)_{p, T, x_{j}} \cdot\left(\frac{\partial x_{i}}{\partial n_{k}}\right)_{n_{j}}
$$

From Eq. (7) it follows for the total differential of $x_{k}$ :

$$
d x_{k}=\frac{d n_{k}}{\sum_{i=1}^{N} n_{i}}-\frac{x_{k}}{\sum_{i=1}^{N} n_{i}} \cdot d \sum_{i=1}^{N} n_{i}
$$

Therefore:

$$
\begin{gathered}
\left(\frac{\partial x_{i}}{\partial n_{k}}\right)_{n_{j}}=-\frac{x_{i}}{\sum_{i=1}^{N} n_{i}} \\
\left(\frac{\partial x_{k}}{\partial n_{k}}\right)_{n_{j}}=\frac{1-x_{k}}{\sum_{i=1}^{N} n_{i}}
\end{gathered}
$$

By equating the functional dependence of Eq. (3) with that of Eq. (9) it is assumed:

$$
G_{R K}^{E} \stackrel{?}{=} G^{E}
$$

Strictly, this assumption that Redlich and Kister needed to utilize in their course of derivation is subject to confirmation, which is why the equality sign in Eq. (15) is supplemented by a question mark. To which extent this kind of questioning is really justified will be given due consideration below.
By invoking Eqs. (6), (13), (14) as well as (15), relationship (11) can be rewritten in the form:

$$
\mu_{k}^{E} \stackrel{?}{=} G_{R K}^{E}-\sum_{i=1}^{N} x_{i}\left(\frac{\partial G_{R K}^{E}}{\partial x_{i}}\right)_{p, T, x_{j}}+\left(\frac{\partial G_{R K}^{E}}{\partial x_{k}}\right)_{p, T, x_{j}}
$$

Eq. (16) is identical with the relationship first published by Redlich and Kister [1]. Since in Eq. (15) it is controversial to equate the quantities $G_{R K}^{E}$ and $G^{E}$ and since Eq. (16) is based on that controversial relationship, uncertainty about the validity of the equality sign is likewise maintained in Eq. (16).

The particularity of Eq. (16) lies in the partial derivatives that require the differentiation to be carried out with respect to the mole fraction of a definite component while the mole fractions of all remaining components are held constant. As recently pointed out [2] this mathematical operation appears to be in contrast to the thermodynamicsrelated interdependence of all mole fractions according to Eq. (8).

Obviously independent of Redlich and Kister, Brown [3] later derived the same equation as that given by Eq. (16). Rowlinson [4] (p. 115) referred to Brown and propagated this relationship as the most convenient way of determining an expression for the excess chemical potential of one of the components from the excess Gibbs free energy of the whole system. The same view has been kept in the latest edition of Rowlinson's book [5] (p. 90). Eq. (16) is also met in van Ness and Abbott's treatises on thermodynamics of nonelectrolyte solutions ([6], p. 90; [7], p. 47), even though the authors recognized the above-mentioned conflict between thermodynamics and mathematics. Nevertheless, all of these authors preferred to make use of Eq. (16) instead of or in addition to the alternative relationship that goes back to Haase [8] and that really reconciles thermodynamics with mathematics. Implemented in computer programs, until now Redlich and Kister's relationship and the pertaining polynomial have a thousand times served as a basis for the purpose of thermodynamic optimization of phase equilibria in chemical engineering [9] and materials science [10].

\section{4. $g^{E}$ as a Homogeneous Function}

Mathematically, the extensive character of the quantity $g^{E}$ is expressed by the fact that $g^{E}$ obeys the conditions of a homogeneous function of first degree in all mole numbers $n_{i}$. It means that the value of $g^{E}$ is increased by the factor $\alpha$ if each $n_{i}$ is multiplied by the same factor. This is well substantiated by practical experience. Each homogeneous function results in Euler's theorem according to which the function multiplied by the degree of homogeneity is equal to the sum of all products between the variables of the function and its partial derivatives with respect to the same variables. For $g^{E}$ this theorem takes the form as given in Eq. (4).

Provided that both the excess Gibbs free energy in the initial state, i.e. $g^{E}$, and the same quantity in the state after the multiplication of each $n_{i}$ with $\alpha$ are represented by the functions $f_{1}$ and $f_{2}$, respectively, then it holds that:

$$
f_{2}\left(p, T, \mathrm{a} \cdot n_{l}, \ldots, \mathrm{a} \cdot n_{N}\right)=\mathrm{a} \cdot f_{l}\left(p, T, n_{l}, \ldots, n_{N}\right)
$$

Euler's theorem results from Eq. (17), and, conversely, Euler's theorem proves the validity of Eq. (17) and thus gives evidence about the fact that $f_{1}$ is really a homogene 
ous function, in the present case of first degree. This becomes apparent by differentiating Eq. (17):

$$
\begin{aligned}
& \left(\frac{\partial f_{2}}{\partial p}\right)_{T, \alpha n_{i}} d p+\left(\frac{\partial f_{2}}{\partial T}\right)_{p, \alpha n_{i}} d T+\sum_{i=1}^{N}\left(\frac{\partial f_{2}}{\partial\left(\alpha n_{i}\right)}\right)_{p, T, \alpha n_{j}} d\left(\alpha n_{i}\right)= \\
& \alpha \cdot\left[\left(\frac{\partial f_{1}}{\partial p}\right)_{T, n_{i}} d p+\left(\frac{\partial f_{1}}{\partial T}\right)_{p, n_{i}} d T+\sum_{i=1}^{N}\left(\frac{\partial f_{1}}{\partial n_{i}}\right)_{p, T, n_{j}} d n_{i}\right]+f_{1} d \alpha
\end{aligned}
$$

Substitution of:

$$
d\left(\mathrm{a} n_{i}\right)=\mathrm{a} d n_{i}+n_{i} d \mathrm{a}
$$

into Eq. (18) and rearrangement of terms yield:

$$
\begin{aligned}
0 & =\left[\left(\frac{\partial f_{2}}{\partial p}\right)_{T, \alpha n_{i}}-\alpha \cdot\left(\frac{\partial f_{1}}{\partial p}\right)_{T, n_{i}}\right] d p \\
& +\left[\left(\frac{\partial f_{2}}{\partial T}\right)_{p, \alpha n_{i}}-\alpha \cdot\left(\frac{\partial f_{1}}{\partial T}\right)_{p, n_{i}}\right] d T \\
& +\alpha \cdot \sum_{i=1}^{N}\left(\left[\left(\frac{\partial f_{2}}{\partial\left(\alpha n_{i}\right)}\right)_{p, T, \alpha n_{j}}-\left(\frac{\partial f_{1}}{\partial n_{i}}\right)_{p, T, n_{j}}\right] d n_{i}\right) \\
& +\left[\sum_{i=1}^{N} n_{i}\left(\frac{\partial f_{2}}{\partial\left(\alpha n_{i}\right)}\right)_{p, T, \alpha n_{j}}-f_{1}\right] d \alpha
\end{aligned}
$$

As $p, T, \alpha$ and all $n_{i}$ are independent variables, the sum of Eq. (20) is equal to zero only if each of the bracket terms is separately equal to zero. Therefore, two of the relevant conclusions of Eq. (20) are:

$$
\begin{aligned}
& \left(\frac{\partial f_{2}}{\partial\left(\mathrm{o} n_{i}\right)}\right)_{p, T, \mathrm{o} n_{j}}=\left(\frac{\partial f_{1}}{\partial n_{i}}\right)_{p, T, n_{j}} \\
& \sum_{i=1}^{N} n_{i}\left(\frac{\partial f_{2}}{\partial\left(\mathrm{o} n_{i}\right)}\right)_{p, T, \mathrm{o} n_{j}}=f_{1}
\end{aligned}
$$

Consideration of the equivalence between $f_{1}$ and $g^{E}$ as agreed at the beginning and substitution of Eqs. (5) and (21) into Eq. (22) lead to Euler's theorem of Eq. (4). As it becomes obvious from the mathematical approach, the validity of Eqs. (4) and (17) depends on the premise that $\alpha$ is an arbitrary quantity independent of all other variables of the supposed homogeneous function.

\section{Violation of the Premises}

In the following let the preceding considerations repeat by assuming that $\alpha$ is equal to the reciprocal of the sum of all mole numbers:

$$
\alpha=\frac{1}{\sum_{i=1}^{N} n_{i}}
$$

In view of Eq. (23), the shape of $f_{2}$ in relationship (17) changes into the right side of Eq. (9), which is equal to $G_{R K}^{E}$ while $f_{1}$ remains the same as defined in the preceding paragraph, i.e. $g^{E}$. Consequently, it follows from Eq. (2) that the right side of relationship (17) becomes identical with $G^{E}$. As a result, Euler's homogeneous function theorem in the form of Eq. (17) confirms the correctness of Eq. (15) which, however, is only true for the special case that $\alpha$ is defined according to Eq. (23).

It follows from Eq. (23) that:

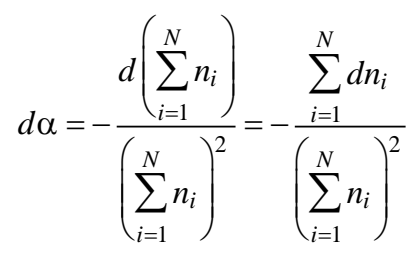

Eq. (24) means that the factor $\alpha$ becomes a function of $n_{i}$ and, hence, loses its status as an independent variable, which in the preceding paragraph was recognized to be the prerequisite for the validity of Euler's homogeneous function theorem. Is that theorem no longer valid, Eq. (17) is not obeyed and neither are Eqs. (21) and (22). Much more important for the validity of the Redlich-Kister approach is that Eq. (15) also loses its justification, inasmuch as Eq. (15) is the consequence of Eq. (17). With Eq. (15) being controversial, the Redlich-Kister relationship of Eq. (16) becomes questionable as well.

There is another striking argument against the validity of the Redlich-Kister relationship. Let Eq. (17) be tentatively assumed to remain valid until further notice and let this be expressed by labeling all of the following equality signs by a question mark. Then, with $\alpha$ according to Eq. (23) and with the total differential of $\alpha$ according to Eq. (24), Eq. (20) converts into:

$$
\begin{aligned}
0 \stackrel{?}{=} & {\left[\left(\frac{\partial f_{2}}{\partial p}\right)_{T, \alpha n_{i}}-\alpha \cdot\left(\frac{\partial f_{1}}{\partial p}\right)_{T, n_{i}}\right] d p } \\
& +\left[\left(\frac{\partial f_{2}}{\partial T}\right)_{p, \alpha n_{i}}-\alpha \cdot\left(\frac{\partial f_{1}}{\partial T}\right)_{p, n_{i}}\right] d T \\
& +\alpha \cdot \sum_{i=1}^{N}\left(\left[\left(\frac{\partial f_{2}}{\partial\left(\alpha n_{i}\right)}\right)_{p, T, \alpha n_{j}}-\left(\frac{\partial f_{1}}{\partial n_{i}}\right)_{p, T, n_{j}}\right.\right. \\
& \left.\left.+\alpha \cdot f_{1}-\alpha \cdot \sum_{i=1}^{N} n_{i}\left(\frac{\partial f_{2}}{\partial\left(\alpha n_{i}\right)}\right)_{p, T, \alpha n_{j}}\right] d n_{i}\right)
\end{aligned}
$$

Since $p, T$ and all $n_{i}$ are independent of each other, Eq. (25) is fulfilled only if each bracket term is equal to zero. Therefore:

$$
\begin{aligned}
& 0 \stackrel{?}{=}\left(\frac{\partial f_{2}}{\partial\left(\alpha n_{i}\right)}\right)_{p, T, \alpha n_{j}}-\left(\frac{\partial f_{1}}{\partial n_{i}}\right)_{p, T, n_{j}} \\
& \quad+\alpha \cdot f_{1}-\alpha \cdot \sum_{i=1}^{N} n_{i}\left(\frac{\partial f_{2}}{\partial\left(\alpha n_{i}\right)}\right)_{p, T, \alpha n_{j}}
\end{aligned}
$$

By invoking Eqs. (5) and (23) and by equating, respectively, $f_{1}$ with $g^{E}, f_{2}$ with $G_{R K}^{E}$ and $\partial\left(\mathrm{a} n_{i}\right)$ with $\partial x_{i}$, it follows from Eq. (26): 
$g^{E} \stackrel{?}{=} \sum_{i=1}^{N} n_{i}\left(\frac{\partial G_{R K}^{E}}{\partial x_{i}}\right)_{p, T, x_{j}}+\sum_{i=1}^{N} n_{i} \cdot\left(\mathrm{m}_{k}^{E}-\left(\frac{\partial G_{R K}^{E}}{\partial x_{k}}\right)_{p, T, x_{j}}\right)$

In view of Eqs. (2) and (15), the rearrangement of terms yields that Eq. (27) becomes identical with Eq. (16) by Redlich and Kister. In other words, Redlich and Kister's relationship is the consequence of mathematical operations that under normal circumstances would prove $g^{E}$ to be a homogeneous function of first degree in all mole numbers. However, these circumstances are not given in the present case. The conditions under which the mathematical operations are applied invalidate the premises underlying the proof for the existence of a homogeneous function and, thus, nullify the results based on these operations. Therefore, in all preceding equations the equality signs labeled by a question mark are false implying that the relationship of Redlich and Kister between the excess chemical potential and the molar excess Gibbs free energy has no mathematical justification.

If, in order to exemplify the implications of the above statement, Eq. (17) were assumed to be a homogeneous function even for $\alpha$ according to Eq. (23), then Eq. (9) would have been valid and, moreover, Eqs. (21) and (22) should have been obeyed under the same conditions as discussed before, i.e. equating $f_{1}$ with $g^{E}, f_{2}$ with $G_{R K}^{E}$ and $\partial\left(\mathrm{a} n_{i}\right)$ with $\partial x_{i}$. In this case, it would follow from Eq. (21) together with Eq. (5) that:

$\mu_{k}^{E} \stackrel{?}{=}\left(\frac{\partial G_{R K}^{E}}{\partial x_{k}}\right)_{p, T, x_{j}}$

The preceding relationship is undoubtedly at variance with the definition of the chemical potential and, thus, at variance with an elementary aspect of chemical thermodynamics.

\section{Practical Consequences}

Inasmuch as Eq. (15) is based on the validity of Eq. (17), which is no longer fulfilled if $\alpha$ obeys Eq. (23), Eq. (15) is wrong and, hence, the identity between $G_{R K}^{E}$ and $G^{E}$ is an incorrect presumption. Consequently, any consideration that proceeds from proposition (9) and tacitly assumes this equation to be obeyed is not justified and leads to inappropriate inferences. One of these inferences is the equivalence recently demonstrated [2] between Redlich and Kister's relationship and those of other authors insofar as the proof of equivalence is based on Eq. (9). It is logical that if the basis of the equivalence is invalid, the same is true for the equivalence itself.

Another inference is more far-reaching with respect to fundamental relevance as the formulation of Eq. (9) can be found in modern thermodynamics textbooks (cf. [11]), p. 319; Eq. (9-6)) and elsewhere in the literature as a basis for thermodynamic calculations. Eq. (9) is the generalized form of the most-accepted function for the mole fraction dependence of $G^{E}$, which is usually called the Redlich-Kister polynomial $^{1)}$ :

1) The nomenclature used in the literature for the variously truncated power series of Eq. (29) totally misjudges the real
$G_{R K}^{E}=\sum_{g=1}^{N-1} \sum_{h=g+1}^{N} x_{g} \cdot x_{h} \sum_{n=0}^{P} A_{g h n} \cdot\left(x_{h}-x_{g}\right)^{n}$

where $x_{g}$ and $x_{h}$ stand for all possible pairs of mole fractions in a system of $N$ components, $A_{g h n}$ are the coefficients and $(\mathrm{P}+2)$ is the degree of the polynomial.

The problem with Eq. (29) is that it strictly meets thermodynamic requirements only after taking Eq. (8) into account. In other words, the polynomial has to be recast in such a way that the mole fraction of the $k$-th component of the system is substituted by the mole fractions of all remaining components. As a result, Eq. (29) changes its appearance and takes the following form:

authorship. Redlich and Kister [1] themselves did never make a secret of the fact that they adopted this polynomial point by point from others, namely from Wohl [12]. Wohl for his part had correctly referred to the empirical ansatz of Margules [13] and to the work of van Laar [14] who had given an approximate theoretical confirmation of Margules's formula. While these authors had only focused on the mole fraction dependence of the vapor pressure over liquid mixtures, the power series concept was later generalized in terms of the excess Gibbs free energy of arbitrary mixtures and was successively advanced by adding new coefficients to the polynomial or by omitting or rearranging some of them selectively (e.g. $[12 ; 15 ; 16])$. It is in the nature of such a power series that, in fact, the accuracy of numerical description changes due to the manner of truncation, nevertheless, the mathematical essence of the approach always remains the same. Therefore it is not surprising when Haase [17] (p. 360) showed that the polynomial nowadays ascribed to Redlich and Kister is completely identical with the conventional ansatz of Margules. The same was later underlined by others ([7], p. 225; [18]). This very fact throws a questionable light on the authorship of Redlich and Kister.

Moreover, Scatchard [19] pointed out that the RedlichKister polynomial is the same as what Guggenheim [20] had proposed before. The new quality inherent in Guggenheim's approach compared to that of Margules was due to a comparatively broader scope and a slightly higher degree of theoretical foundation. Guggenheim proceeded from the simplest possible form of an empirical power series for an energy term of an arbitrary mixture rather than merely for the fugacity coefficient of a vapour, as Margules had done. Strictly, Guggenheim concentrated on that part of the total Gibbs free energy of any mixture that causes this mixture to deviate from Raoult's law, for which later the term excess function was coined. By customizing the questionable energy term in such a way that it represents a homogeneous function of first degree in the mole numbers, Guggenheim ensured that the empirical mathematical function meets the same fundamental thermodynamic requirements as any other thermodynamic state function does. For the above-mentioned reasons and in order to acknowledge the true value of the contributions to the field it is more appropriate to associate the polynomial with the authorship of Margules and Guggenheim rather than Redlich and Kister. 


$$
\begin{aligned}
& G^{E}=\sum_{\substack{g=1 \\
\neq k}}^{N-1} \sum_{\substack{h=g+1 \\
\neq k}}^{N} x_{g} \cdot x_{h} \sum_{n=0}^{P} A_{g h n} \cdot\left(x_{h}-x_{g}\right)^{n} \\
& +\sum_{h=k+1}^{N}\left(1-x_{h}-\sum_{\substack{m=1 \\
\neq h, k}}^{N} x_{m}\right) \cdot x_{h} \sum_{n=0}^{P} A_{k h n} \cdot\left(2 x_{h}+\sum_{\substack{m=1 \\
\neq h, k}}^{N} x_{m}-1\right)^{n} \\
& +\sum_{g=1}^{k-1} x_{g} \cdot\left(1-x_{g}-\sum_{\substack{m=1 \\
\neq g, k}}^{N} x_{m}\right) \sum_{n=0}^{P} A_{g k n} \cdot\left(-2 x_{g}-\sum_{\substack{m=1 \\
\neq g, k}}^{N} x_{m}+1\right)^{n}
\end{aligned}
$$

Interestingly, after having transformed the thermodynamically inadequate representation of Eq. (29) into Eq. (30) the application of Redlich and Kister's $\mathrm{m}_{k}^{E}$ vs. $G^{E}$ relationship according to Eq. (16) becomes impossible from mathematical point of view since the conditions required for the validity of the partial derivatives of Eq. (16) are no longer fulfilled by the formulation of Eq. (30). Therefore, in order to derive from Eq. (30) an analytical expression for the mole fraction dependence of the excess chemical potential, the only applicable $\mathrm{m}_{k}^{E}$ vs. $G^{E}$ relationship is the one of Haase ([8]; see also [2]). Haase's relationship reads:

$$
\mu_{k}^{E}=G^{E}-\sum_{\substack{i=1 \\ \neq \neq}}^{N} x_{i}\left(\frac{\partial G^{E}}{\partial x_{i}}\right)_{p, T, x_{j \neq k}}
$$

Application of Eq. (31) to the expression of $G^{E}$ according to Eq. (30) delivers:

$$
\begin{aligned}
\mu_{k}^{E}= & \left(\sum_{g=1}^{k-1} x_{g} \sum_{n=0}^{P} A_{g k n} \cdot\left(x_{k}-x_{g}\right)^{n}\right) \cdot\left(1-x_{k}\right) \\
& +\sum_{h=k+1}^{N} x_{h} \sum_{n=0}^{P} A_{k h n} \cdot\left(x_{h}-x_{k}\right)^{n} \\
& -\sum_{i=2}^{N} x_{i} \sum_{g=1}^{i-1} x_{g} \sum_{n=0}^{P} A_{g i n} \cdot\left(x_{i}-x_{g}\right)^{n} \\
& +\sum_{g=1}^{k-1} x_{g} \sum_{n=1}^{P} n A_{g k n} \cdot\left(x_{k}-x_{g}\right)^{n-1} \\
& \left.-\sum_{h=k+1}^{N} x_{h} \sum_{n=1}^{P} n A_{k h n} \cdot\left(x_{h}-x_{k}\right)^{n-1}\right)^{\prime}\left(1-x_{k}\right) \\
& -\sum_{i=1}^{N} x_{i}^{2} \cdot\left(\sum_{g=1}^{i-1} x_{g} \sum_{n=1}^{P} n A_{g i n} \cdot\left(x_{i}-x_{g}\right)^{n-1}\right. \\
& \neq k \\
& \left.-\sum_{h=i+1}^{N} x_{h} \sum_{n=1}^{P} n A_{i h n} \cdot\left(x_{h}-x_{i}\right)^{n-1}\right)
\end{aligned}
$$

Strangely enough, Eq. (16) although being incorrect in the sense described above yields the same analytical expression as Eq. (32) if applied to the thermodynamically inadequate formulation of Eq. (29). This has to do with the mathematical feature of Eq. (29). As remarkable as this point is with respect to practical implementation, it does not detract from the fact that Redlich and Kister's relationship and the pertaining polynomial contradict fundamental principles of chemical thermodynamics and mathematics.

Regarding these principles the most important consequence of the present investigation is the non-existent justification of Eq. (15) which makes Eq. (9) a thermodynami- cally irrelevant formulation. As it becomes apparent above, the only reason why Eq. (9) and its representation by Eq. (29) get in conflict with fundamental principles is that they both ignore Eq. (8), with Eq. (8) expressing a purely thermodynamic fact. Indeed, without any thermodynamic background knowledge nothing could be objected to Eq. (9) from mathematical point of view. The same is true as to the premises for the validity of Euler's homogeneous function theorem by introducing the factor $\alpha$ according to Eq. (23). Thus, it is a thermodynamic aspect that causes mathematical rules to be violated and not the other way around. A correct mathematical operation will not necessarily yield a mathematically incorrect result by merely disobeying thermodynamic principles. Therefore, Eq. (16), despite contradicting thermodynamic facts, results in the mathematically correct expression of Eq. (32), if it is applied to the thermodynamically inadequate polynomial of Eq. (29). That Eq. (32), apart from being mathematically correct, additionally proves to be correct from thermodynamic point of view can only be demonstrated and is demonstrated above by applying Haase's thermodynamically consistent $\mathrm{m}_{k}^{E}$ vs. $G^{E}$ relationship to the polynomial form of Eq. (30).

Considering the significance that the Redlich-Kister polynomial has in chemical thermodynamics, the analysis described above requires to recast this polynomial into a thermodynamically compatible form. That means, the representation of Eq. (29) has to be replaced by Eq. (30) for which the term Margules-Guggenheim polynomial is proposed. The Margules-Guggenheim polynomial in its turn is inevitably incompatible with the questionable $\mathrm{m}_{k}^{E}$ vs. $G^{E}$ relationship of Redlich and Kister and, thus, the circle closes. The paradoxical situation outlined at the beginning, with mathematical assumptions contradicting thermodynamic reality, is ultimately due to non-strictly taking account of thermodynamic reality in one important respect, i.e. the definition of what has so far been called the Redlich-Kister polynomial.

\section{Conclusions}

Mathematically the relationship of Redlich and Kister results from a special case of treating the excess Gibbs free energy of a multicomponent mixture as a homogeneous function of first degree in the mole numbers due to which the mole numbers are transformed into mole fractions. The consequence of this particular kind of transformation is that the premises for the existence of a homogeneous function become violated so that the outcome and the related inferences lose mathematical justification. The thermodynamic inconsistency of Redlich and Kister's $\mathrm{m}_{k}^{E}$ vs. $G^{E}$ relationship has its equivalent in the thermodynamically inadequate representation of the Redlich-Kister polynomial.

\section{References}

[1] Redlich, O., Kister, A. T. Algebraic Representation of Thermodynamic Properties and the Classification of Solutions. Industr. Eng. Chem. 40, 345-348, 1948.

[2] Näfe, H. Relationship between the Partial Molar and Molar Quantity of a Thermodynamic State Function in a Multicomponent Mixture - Revisited. J. Chem. Thermod. 61, 138-145, 2013.

[3] Brown, W. B. The Statistical Thermodynamics of Mixtures of Lennard-Jones Molecules. I. Random Mixtures. Phil. Trans. A250, 175-220, 1957.

[4] Rowlinson, J. S. Liquids and Liquid Mixtures, London: Butterworths Scientific Publ., 1959. 
[5] Rowlinson, J. S., Swinton, F. L. Liquids and Liquid Mixtures, London-Boston-Sydney-Wellington-Durban-Toronto: Butterworths Scientific, 1982.

[6] van Ness, H. C. Classical Thermodynamics of NonElectrolyte Solutions, Oxford-London-New YorkParis: Pergamon Press, 1964.

[7] van Ness, H. C., Abbott, M. M. Classical Thermodynamics of Nonelectrolyte Solutions with Applications to Phase Equilibria, New York-St. Louis-San Francisco-Sydney-Tokyo-Toronto: McGraw-Hill Book Co., 1982.

[8] Haase, R. Einige allgemeine Beziehungen für $\mathrm{Zu}$ standsfunktionen bei Vielkomponentensystemen. $Z$. Naturforschg. 3a, 285-290, 1948.

[9] Prausnitz, J. M., Lichtenthaler, R. N., de Azevedo, E. G. Molecular Thermodynamics of Fluid-Phase Equilibria, (3rd ed.). Upper Saddle River, NJ: Prentice Hall PTR, 1999.

[10] Hillert, M. Phase Equilibria, Phase Diagrams and Phase Transformations. Their Thermodynamic Basis, Cambridge: Cambridge University Press, 1998.

[11] Tester, J. W., Modell, M. Thermodynamics and Its Applications. Upper Saddle River, NJ: Prentice Hall PTR, 1997.
[12] Wohl, K. Thermodynamic Evaluation of Binary and Ternary Liquid Systems. Trans. Am. Inst. Chem. Eng. 42, 215-249, 1946.

[13] Margules, M. Über die Zusammensetzung der gesättigten Dämpfe von Mischungen. Sitzb. Akad. Wiss. Wien, Math.-naturwiss. Kl., Abt. 2 a 104, 1243-1278, 1985.

[14] van Laar, J. J. Über Dampfspannungen von binären Gemischen. Z. phys. Chem. 72, 723-751, 1910.

[15] Benedict, M., Johnson, C. A., Solomon, E., Rubin, L. C. Extractive and Azeotropic Distillation. II. Separation of Toluene from Paraffins by Azeotropic Distillation with Methanol. Trans. Am. Inst. Chem. Eng. 41, 371-392, 1945.

[16] Scatchard, G., Hamer, W. J. The Application of Equations for the Chemical Potentials to Partially Miscible Solutions. J. Am. Chem. Soc. 57, 1805-1809, 1935.

[17] Haase, R. Thermodynamik der Mischphasen, BerlinGöttingen-Heidelberg: Springer-Verlag, 1956.

[18] Gokcen, N. A. Gibbs-Duhem-Margules Laws. J. Phase Equilibria 17, 50-51, 1996.

[19] Scatchard, G. Equilibrium in Non-Electrolyte Mixtures. Chem. Rev. 44, 7-35, 1949.

[20] Guggenheim, E. A. The Theoretical Basis of Raoult's Law. Trans. Faraday Soc. 33, 151-156, 1937. 